\title{
Motion of heavy particles coupled to fermionic and bosonic environments in one dimension
}

\author{
A. O. Caldeira ${ }^{1, *}$ and A. H. Castro Neto $^{2}$ \\ 1 Department of Physics, University of Illinois at Urbana-Champaign \\ 1110 W.Green St., Urbana, IL, 61801-3080 \\ 2 Institute for Theoretical Physics \\ University of California \\ Santa Barbara, CA, 93106-4030
}

\begin{abstract}
Making use of a simple unitary transformation we change the hamiltonian of a particle coupled to an one dimensional gas of bosons or fermions to a new form from which the many body degrees of freedom can be easily traced out. The effective dynamics of the particle allows us to compute its damping constant in terms of the reflection coefficient of the interaction potential and the occupation number of the environmental particles. We apply our results to a delta repulsive potential.

PACS numbers: 05.30.Fk, 05.30.Jp, 05.40.+j
\end{abstract}

Typeset using REVTEX 


\section{INTRODUCTION}

When one wants to study the quantum dynamics of a non-isolated system a possible way to attack the problem is to assume that the system we want to study is coupled to a bath of harmonic oscillators with a specific spectral function [1, 2] . When this is done we are not interested in the microscopic details of the interaction of the system with its environment but rather in those essential features that help us to recover the phenomenological equations of motion in the classical limit. This has been the approach used to study dissipative effects in the phenomena of macroscopic quantum tunneling [2] and coherence [3].

A second approach to investigate the quantum dynamics of a system interacting with its environment would be to treat the problem from a microscopic point of view. In this case there are no standard expressions for the effective dynamics of the system of interest and for each problem they must be developed from the microscopic equations according to the appropriate approximations for the particular interaction we are dealing with. There are very important problems which are suitable for the microscopic approach, such as; motion of ions in normal [4.5] and superfluid $\mathrm{He}^{3}$ [5], mobility of particles in metals [6-8] and diffusion of heavy particles in solids [9], to name just a few.

Actually we could also incorporate in the microscopic approach the recently developed formulation for dealing with mobility and diffusion of quantum solitons in problems of coupled [10] or non-linear field theories [11]. Here, the collective coordinate method [12] is shown to lead us to another very simple model [13] for describing quantum dissipation.

In this paper we have shown that the hamiltonian of a particle coupled to bosons and fermions in one dimension can also be mapped into the model of ref. [13]. In this case it is simply done through the application of a unitary operator which uniformly translates the positions of all the environmental particles changing the origin of their coordinate frame to the position of the external particle. As a matter of fact this procedure is valid for any interaction potential which depends only on the distance between the particle of interest and each of the environmental particles. At the end we specialize on the delta repulsive 
potential because of mathematical convenience and transparence to analyze the effects of foward and backward scattering processes.

We shall start by applying the above-mentioned unitary transformation to the original hamiltonian and showing how its transformed version can be used in the well-known Feynman-Vernon approach [1, [14] for dealing with the reduced density operator of the external particle. This is done in section II.

In section III we briefly review the coherent state representation for the density operator of the system and obtain the real time reduced density operator of the particle from which

we can get its damping constant. Although this has been presented in another paper 10 we decided to include it here to make this contribution more self-contained and to correct some of our previous expressions.

In section IV we analyze the expression for the damping constant and obtain its temperature dependence in the case of bosonic and fermionic environments and finally present our conclusions in section $\mathrm{V}$. We have also included an appendix where we discuss the approach in thermal equilibrium and the approximations made in our calculations.

\section{THE CANONICAL TRANSFORMATION}

Let us start by describing the model that will be used throughout this paper. We assume that a particle of mass $M$ is interacting with an one dimensional gas of $N$ fermions or bosons of mass $m$ through the hamiltonian

$$
H=\frac{p^{2}}{2 M}+\sum_{i=1}^{N} U\left(q-q_{i}\right)+\sum_{i=1}^{N} \frac{p_{i}^{2}}{2 m}
$$

where $p, q, p_{i}$, and $q_{i}$ are respectively the momenta and positions of the heavy particle and of the $i^{t h}$ environmental particle and $U\left(q-q_{i}\right)$ can, in principle, be any interaction potential. We shall only assume it has a delta repulsive form later on in this work.

The dynamics of the particle is governed by its reduced density operator which is given by [1, 14] 


$$
\tilde{\rho}(x, y, t)=\int d q_{1} d q_{2} \ldots d q_{N}\left\langle x, q_{1}, \ldots, q_{N}\left|e^{i \frac{H t}{\hbar}} \rho(0) e^{-i \frac{H t}{\hbar}}\right| y, q_{1}, \ldots, q_{N}\right\rangle
$$

where $\rho(0)$ is the initial density operator of the whole system.

Now, introducing the unitary operator $U$

$$
U=e^{-\frac{i}{\hbar} \sum_{j=1}^{N} p_{j} q}
$$

we can use the identity $U^{-1} U=1$ between any two operators in (2.2) to get

$$
\tilde{\rho}(x, y, t)=\int d q_{1} d q_{2} \ldots d q_{N}\left\langle x, q_{1}, \ldots, q_{N}\left|U^{-1} e^{i \frac{H^{\prime} t}{\hbar}} \rho^{\prime}(0) e^{-i \frac{H^{\prime} t}{\hbar}} U\right| y, q_{1}, \ldots, q_{N}\right\rangle
$$

where $H^{\prime}$ is the transformed hamiltonian [15]

$$
H^{\prime}=U H U^{-1}=\frac{1}{2 M}\left(p-\sum_{j=1}^{N} p_{j}\right)^{2}+\sum_{j=1}^{N}\left(\frac{p_{j}^{2}}{2 m}+U\left(q_{j}\right)\right)
$$

and $\rho^{\prime}(0)=U \rho(0) U^{-1}$ is the transformed initial condition which we analyze below.

It should be noticed that the application of the unitary transformation $U$ to the hamiltonian $H$ has modified it in such a way that now the external particle interacts via momentummomentum coupling with a set of particles which are scattered by a potential $U(q)$. The same transformation was used by Castella and Zotos [16] for the exact diagonalization of the problem via the Bethe ansatz.

Now, inserting the closure relation

$$
\int d x d q_{1} d q_{2} \ldots d q_{N}\left|x, q_{1}, \ldots, q_{N}\right\rangle\left\langle x, q_{1}, \ldots, q_{N}\right|=1
$$

twice between the unitary operators $U$ and $U^{-1}$ and the transformed density operator $\rho^{\prime}(t)$ one has

$$
\begin{array}{r}
\tilde{\rho}(x, y, t)=\int d q_{1} d q_{2} \ldots d q_{N} d r_{1} d r_{2} \ldots d r_{N}\left\langle r_{1}, \ldots, r_{N}\left|e^{-\frac{i}{\hbar} \sum_{j=1}^{N} p_{j}(x-y)}\right| q_{1}, \ldots, q_{N}\right\rangle \\
\left\langle x, q_{1}, \ldots, q_{N}\left|\rho^{\prime}(t)\right| y, r_{1}, \ldots, r_{N}\right\rangle
\end{array}
$$

which is more cumbersome than (2.2) due to the existence of the first term on its r.h.s.. This complication was brought about by the unitary transformation and reflects the fact 
that after solving the dynamics in the more appropriate representation we need to return to the original representation from where we started.

Nevertheless things are not so awkward as they seem. Since we are most of the time, and particularly in this work, interested in the average values of observables such as $\langle x(t)\rangle$, $\langle p(t)\rangle,\left\langle x^{2}(t)\right\rangle$ or $\left\langle p^{2}(t)\right\rangle$ we do not really need (2.6) as it stands but rather its diagonal elements only.

The justification for this assertion for observables which are only $\mathrm{x}$-dependent is trivial because

$$
\left\langle x^{n}(t)\right\rangle=\int d x x^{n} \tilde{\rho}(x, x, t)
$$

and the first term on the r.h.s. of (2.6) becomes $\delta\left(q_{1}-r_{1}\right) \ldots \delta\left(q_{N}-r_{N}\right)$ which can be trivially integrated yielding

$$
\left\langle x^{n}(t)\right\rangle=\int d x d q_{1} \ldots d q_{N} x^{n}\left\langle x, q_{1}, \ldots, q_{N}\left|\rho^{\prime}(t)\right| x, q_{1}, \ldots, q_{N}\right\rangle
$$

For p-dependent operators the demonstration is also easy although not immediate as in (2.7-2.8). Now, what we need is

$$
\langle p(t)\rangle=\operatorname{Lim}_{x \rightarrow y} \frac{\hbar}{2 i}\left(\frac{d}{d x}-\frac{d}{d y}\right) \tilde{\rho}(x, y, t)
$$

which upon the application of the derivatives to (2.6) yields

$$
\begin{array}{r}
\langle p(t)\rangle=\frac{\hbar}{2 i} \operatorname{Lim}_{x \rightarrow y} \int d q_{1} d q_{2} \ldots d q_{N} d r_{1} d r_{2} \ldots d r_{N}\left\langle r_{1}, \ldots, r_{N}\left|e^{-\frac{i}{\hbar} \sum_{j=1}^{N} p_{j}(x-y)}\right| q_{1}, \ldots, q_{N}\right\rangle \\
\left(\frac{d}{d x}-\frac{d}{d y}\right)\left\langle x, q_{1}, \ldots, q_{N}\left|\rho^{\prime}(t)\right| y, r_{1}, \ldots, r_{N}\right\rangle .
\end{array}
$$

In the limit $x \rightarrow y$ the first term of the integrand above becomes once again $\delta\left(q_{1}-r_{1}\right) \ldots \delta\left(q_{N}-\right.$ $\left.r_{N}\right)$ which can be integrated to give

$$
\langle p(t)\rangle=\frac{\hbar}{2 i} \operatorname{Lim}_{x \rightarrow y} \int d q_{1} d q_{2} \ldots d q_{N}\left(\frac{d}{d x}-\frac{d}{d y}\right)\left\langle x, q_{1}, \ldots, q_{N}\left|\rho^{\prime}(t)\right| y, q_{1}, \ldots, q_{N}\right\rangle
$$

and so, what we really need in both cases is,

$$
\tilde{\rho}^{\prime}(x, y, t)=\int d q_{1} d q_{2} \ldots d q_{N}\left\langle x, q_{1}, \ldots, q_{N}\left|\rho^{\prime}(t)\right| y, q_{1}, \ldots, q_{N}\right\rangle
$$


Here one should notice that this result is valid only if we want to compute the abovementioned averages. Were we interested in the time evolution of $\tilde{\rho}(x, y, t)$ this statement would not be valid.

Before leaving this section, let us try to clarify the choice we will make for the initial condition $\rho^{\prime}(0)$ in this work. As we have seen from $(2.4) \rho^{\prime}(0)$ can be prepared in terms of the eigenstates of the transformed hamiltonian (2.5). Our particular choice will be to assume that the particle is in a pure state (a wave packet centered at the origin) and the environment is in equilibrium in the presence of the particle. This means we can write $\rho^{\prime}(0)$ as

$$
\rho^{\prime}(0)=\rho_{s} \rho_{e q}^{\prime}=\rho_{s} e^{-\beta H_{e}}
$$

where $\rho_{s}$ refers only to the particle and $H_{e}$ is given by

$$
H_{e}=\sum_{j=1}^{N}\left(\frac{p_{j}^{2}}{2 m}+U\left(q_{j}\right)\right)
$$

Notice that this is only valid as long as one neglects the spread of the initial wave packet about the origin when preparing the initial state of the composite system.

\section{THE FEYNMAN-VERNON APPROACH}

Having specified the central object of this paper we should now choose the most appropriate representation in which we should write it. Since our environment is composed of indistinguishable particles (bosons or fermions) it is convenient to write the transformed hamiltonian (2.5) in its second quantized version which reads 17

$$
H^{\prime}=\frac{1}{2 M}\left(p-\sum_{i, j} \hbar g_{i j} a_{i}^{\dagger} a_{j}\right)^{2}+\sum_{i}\left(\hbar \Omega_{i}-\mu\right) a_{i}^{\dagger} a_{i}
$$

where $g_{i j}=\frac{1}{\hbar}\left\langle i\left|p^{\prime}\right| j\right\rangle$ is the matrix element of the momentum operator of a single environment particle between eingenstates $|i\rangle$ and $|j\rangle$ of $H_{e}$ in (2.14), $\Omega_{i}$ are the eigenfrequencies of these states and $\mu$ is the chemical potential. The operators $a_{i}$ and $a_{i}^{\dagger}$ are the standard annihilation and creation operators for bosons or fermions. 
Then we can use the initial conditions (2.13) in (2.12) to write the standard FeynmanVernon expression [1, 14]

$$
\tilde{\rho}^{\prime}(x, y, t)=\int d x^{\prime} \int d y^{\prime} \mathcal{J}\left(x, y, t ; x^{\prime}, y^{\prime}, 0\right) \rho_{s}\left(x^{\prime}, y^{\prime}, 0\right)
$$

where

$$
\begin{array}{r}
\mathcal{J}\left(x, y, t ; x^{\prime}, y^{\prime}, 0\right)=\int d q_{1} \ldots d q_{N} d r_{1} \ldots d r_{N} d s_{1} . . d s_{N}\left\langle x, q_{1}, \ldots, q_{N}\left|e^{-\frac{i}{\hbar} H^{\prime} t}\right| x^{\prime}, r_{1}, \ldots, r_{N}\right\rangle \\
\left\langle r_{1}, \ldots, r_{N}\left|e^{-\beta H_{e}}\right| s_{1}, \ldots, s_{N}\right\rangle\left\langle y^{\prime}, s_{1}, \ldots, s_{N}\left|e^{\frac{i}{\hbar} H^{\prime} t}\right| y, q_{1}, \ldots, q_{N}\right\rangle .
\end{array}
$$

However, as we have chosen to represent our hamiltonian $H^{\prime}$ in its second quantized form we had better perform all the integrals in (3.3) in the coherent state representation for the creation and annihilation operators 18 involved therein. In doing so we can rewrite $\mathcal{J}$ in (3.3) as

$$
\mathcal{J}\left(x, y, t ; x^{\prime}, y^{\prime}, 0\right)=\int \frac{d^{2} \vec{\alpha}}{\pi} \frac{d^{2} \vec{\gamma}}{\pi} \frac{d^{2} \vec{\delta}}{\pi}\left\langle x, \vec{\alpha}\left|e^{-\frac{i}{\hbar} H^{\prime} t}\right| x^{\prime}, \vec{\gamma}\right\rangle\left\langle\vec{\gamma}\left|e^{-\beta H_{e}}\right| \vec{\delta}\right\rangle\left\langle y^{\prime}, \vec{\delta}\left|e^{\frac{i}{\hbar} H^{\prime} t}\right| y, \vec{\alpha}\right\rangle
$$

where $\vec{\alpha}$ is a complex vector with infinte number of components and $\frac{d^{2} \vec{\alpha}}{\pi}=$ $\frac{1}{\pi} \prod_{i} d\left(\operatorname{Re} \alpha_{i}\right) d\left(\operatorname{Im} \alpha_{i}\right)$. Equation (3.4) can be written in a more appropriate form if we use the functional integral representation for all the time evolution operators (in real and imaginary time) of its integrand. We can write

$$
\mathcal{J}\left(x, y, t ; x^{\prime}, y^{\prime}, 0\right)=\int \mathcal{D} x\left(t^{\prime}\right) \mathcal{D} y\left(t^{\prime}\right) e^{\frac{1}{\hbar}\left(S_{0}[x]-S_{0}[y]\right)} \mathcal{F}[x, y]
$$

where

$$
S_{0}[x]=\int_{0}^{t} d t^{\prime} \frac{1}{2} M \dot{x}^{2}\left(t^{\prime}\right)
$$

is the action of the particle if it were not coupled to the environment, $\mathcal{F}[x, y]$, the well-known influence functional, is given by

$$
\mathcal{F}[x, y]=\int \frac{d^{2} \vec{\alpha}}{\pi} \frac{d^{2} \vec{\gamma}}{\pi} \frac{d^{2} \vec{\delta}}{\pi} \rho_{R}\left(\vec{\gamma}^{*}, \vec{\delta}\right) \int_{\vec{\gamma}}^{\alpha^{*}} \mathcal{D}^{2} \vec{\alpha} \int_{\vec{\delta}^{*}}^{\vec{\alpha}} \mathcal{D}^{2} \vec{\delta} e^{\frac{i}{\hbar}\left(S_{I}[x, \vec{\alpha}]-S_{I}^{*}[y, \vec{\delta}]\right)}
$$

with 


$$
S_{I}[x, \vec{\alpha}]=\int_{0}^{t} d t^{\prime}\left(\frac{i \hbar}{2} \sum_{n}\left(\alpha_{n}^{*} \dot{\alpha}_{n}-\alpha_{n} \dot{\alpha}_{n}^{*}\right)+\dot{x} \sum_{m, n} \hbar g_{m, n} \alpha_{m}^{*} \alpha_{n}-\sum_{n}\left(\hbar \Omega_{n}-\mu\right) \alpha_{n}^{*} \alpha_{n}\right)
$$

and

$$
\begin{aligned}
\rho_{R}\left(\vec{\gamma}^{*}, \vec{\delta}\right) & =\frac{\exp \left\{e^{-\beta\left(\hbar \Omega_{n}-\mu\right)} \gamma_{n}^{*} \delta_{n}\right\}}{Z}, \\
Z & =\int \frac{d^{2} \vec{\alpha}}{\pi} \exp \left\{e^{-\beta\left(\hbar \Omega_{n}-\mu\right)}\left|\alpha_{n}\right|^{2}\right\} .
\end{aligned}
$$

The integral in (3.7) is exactly the same that has been evaluated in the context of the polaron dynamics in ref. [10]. The only difference is that we now must deal with the environmental particles as being massive bosons or fermions. The result of the integration follows exactly as in [10] and reads [19]

$$
\mathcal{F}[x, y]=[\operatorname{det}(1 \mp \bar{N} \Gamma[x, y])]^{\mp 1}
$$

where the -1 refers to bosons and +1 refers to fermions, $\bar{N}$ is the ocupation number operator given by

$$
\begin{aligned}
\bar{N}_{i j} & =\delta_{i j} \bar{n}_{i} \\
\bar{n}_{i} & =\frac{1}{e^{\beta\left(\Omega_{i}-\mu\right)} \mp 1}
\end{aligned}
$$

and the functional $\Gamma[x, y]$ is such that

$$
\Gamma_{n m}[x, y]=W_{n m}[x]+W_{n m}^{*}[y]+\sum_{k} W_{n k}^{*}[y] W_{k m}[x]
$$

with $W_{n m}$ satisfying the integral equation

$$
W_{n m}[x, \tau]=\int_{0}^{\tau} d t^{\prime} W_{n m}^{0}\left(\dot{x}, t^{\prime}\right)+\sum_{k} \int_{0}^{\tau} d t^{\prime} \int_{0}^{t^{\prime}} d t^{\prime \prime} W_{n k}^{0}\left(\dot{x}, t^{\prime}\right) W_{k m}\left(\dot{x}, t^{\prime \prime}\right)
$$

where

$$
W_{n m}^{0}\left(t^{\prime}\right)=i g_{n m} \dot{x}\left(t^{\prime}\right) e^{i\left(\Omega_{n}-\Omega_{m}\right) t^{\prime}}\left(1-\delta_{n m}\right) .
$$

So far our results are exact. 
Since the influence functional $\mathcal{F}$ must still be integrated in (3.5) its form (3.10) is useless. In order to evaluate the double functional integral in (3.5) we have to make some approximations in our present expression for $\mathcal{F}[x, y]$. Observing, from (3.13) and (3.14), that $\Gamma[x, y]$ depends on the velocities $\dot{x}$ and $\dot{y}$ we can rely on the assumption that only slow paths will contribute to (3.5) and expand (3.10) up to quadratic terms in $\dot{x}$ and $\dot{y}$.

This was the procedure followed in reference [10] and therefore we shall only quote the final result of this approximation which reads

$$
\mathcal{F}[x, y]=e^{-\frac{i}{\hbar} \Phi_{I}[x, y]} e^{-\frac{1}{\hbar} \Phi_{R}[x, y]}
$$

where

$$
\Phi_{I}=\int_{0}^{t} d t^{\prime} \int_{0}^{t^{\prime}} d t^{\prime \prime}\left(\dot{x}\left(t^{\prime}\right)-\dot{y}\left(t^{\prime}\right)\right) \hbar \Gamma_{I}\left(t^{\prime}-t^{\prime \prime}\right)\left(\dot{x}\left(t^{\prime \prime}\right)+\dot{y}\left(t^{\prime \prime}\right)\right)
$$

and

$$
\Phi_{R}=\int_{0}^{t} d t^{\prime} \int_{0}^{t^{\prime}} d t^{\prime \prime}\left(\dot{x}\left(t^{\prime}\right)-\dot{y}\left(t^{\prime}\right)\right) \hbar \Gamma_{R}\left(t^{\prime}-t^{\prime \prime}\right)\left(\dot{x}\left(t^{\prime \prime}\right)-\dot{y}\left(t^{\prime \prime}\right)\right)
$$

with

$$
\Gamma_{R}(t)=\frac{1}{2} \sum_{i, j}\left|g_{i j}\right|^{2}\left(\bar{n}_{i}+\bar{n}_{j}+2 \bar{n}_{i} \bar{n}_{j}\right) \cos \left(\Omega_{i}-\Omega_{j}\right) t
$$

and

$$
\Gamma_{I}(t)=\sum_{i, j}^{\infty}\left|g_{i j}\right|^{2}\left(\bar{n}_{i}-\bar{n}_{j}\right) \sin \left(\Omega_{i}-\Omega_{j}\right) t
$$

From these expressions we can determine the damping function $\gamma(t)$ as

$$
\gamma(t)=-\frac{\hbar}{M} \frac{d \Gamma_{I}(t)}{d t}=-\frac{\hbar}{2 M} \int d \omega \int d \omega^{\prime} S\left(\omega, \omega^{\prime}\right)\left[\bar{n}(\omega)-\bar{n}\left(\omega^{\prime}\right)\right]\left(\omega-\omega^{\prime}\right) \cos \left(\omega-\omega^{\prime}\right) t
$$

and the diffusion function $D(t)$ as

$$
D(t)=-\hbar \frac{d^{2} \Gamma_{R}}{d t^{2}}=\frac{\hbar^{2}}{2} \int d \omega \int d \omega^{\prime} S\left(\omega, \omega^{\prime}\right)\left[\bar{n}(\omega)+\bar{n}\left(\omega^{\prime}\right)+2 \bar{n}(\omega) \bar{n}\left(\omega^{\prime}\right)\right]\left(\omega-\omega^{\prime}\right)^{2} \cos \left(\omega-\omega^{\prime}\right) t
$$


In the expressions for $\gamma(t)$ and $D(t)$ we have introduced the scattering function $S\left(\omega, \omega^{\prime}\right)$ which we define as

$$
S\left(\omega, \omega^{\prime}\right)=\sum_{i, j}\left|g_{i j}\right|^{2} \delta\left(\omega-\Omega_{i}\right) \delta\left(\omega^{\prime}-\Omega_{j}\right) .
$$

In this paper we shall be mostly concerned with the damping function (3.20).

\section{THE DAMPING CONSTANT}

Having deduced the explicit form of $\gamma(t)$ our next step is to investigate its long time

behavior. As in ref. [10 it is our goal now to evaluate the scattering function $S\left(\omega, \omega^{\prime}\right)$ for a specific choice of our model hamiltonian. Let us assume that the interaction potential between the external body and the environmental particles is given by

$$
U\left(q-q_{i}\right)=V_{0} a \delta\left(q-q_{i}\right)
$$

where $V_{0}$ and $a$ are, respectively, the height and the range of the potential barrier.

Now, because of the unitary transformation we used, the expression of $S\left(\omega, \omega^{\prime}\right)$ depends only on the evaluation of the matrix elements of the single particle momentum operator between two different states in (2.14). As this is a hamiltonian of non-interacting particles subject to a delta repulsive potential at the origin it is a very simple task to obtain its eigenstates. Observe that this problem is essentially the one of an impurity in one dimension that has been studied using many different techniques, from bosonization and renormalization group calculations [20,21] to Bethe ansatz approaches [22].

Let us suppose that our environmental particles are contained in a very large one dimensional box of length $2 L$. It is then easy to show that we have even and odd solutions given by [23],

$$
\begin{aligned}
& u_{E}(k, x)=\frac{1}{\sqrt{L}} \cos \left(k|x|+\delta_{k}\right) \\
& u_{O}(k, x)=\frac{1}{\sqrt{L}} \sin (k x)
\end{aligned}
$$


where

$$
\delta_{k}=\frac{1}{2} \tan ^{-1}\left(\frac{\operatorname{Im}(t+r)}{\operatorname{Re}(t+r)}\right)
$$

with

$$
\begin{aligned}
r(k) & =\frac{i \kappa}{2|k| D(k)} \\
t(k) & =1+r(k) \\
D(k) & =1-\frac{i \kappa}{2|k|} \\
\kappa & =\frac{4 m V_{0}}{\hbar^{2}} .
\end{aligned}
$$

Notice that the phase shifts of the odd solutions are zero and that we can further relate $\delta_{k}$ to the transmission and reflection coefficients of the delta repulsive potential as (the case of a general potential without bound states is treated in the Appendix)

$$
\begin{gathered}
T(k)=|t(k)|^{2}=\cos ^{2} \delta_{k} \\
R(k)=|r(k)|^{2}=\sin ^{2} \delta_{k} .
\end{gathered}
$$

Our first step to get an analytic expression for $S\left(\omega, \omega^{\prime}\right)$ is to evaluate

$$
g_{k k^{\prime}}=\int_{-L}^{L} \frac{d x}{L} u_{E}(k, x)\left(-i \frac{d}{d x} u_{O}\left(k^{\prime}, x\right)\right)
$$

which gives

$$
\begin{aligned}
g_{k k^{\prime}} & =-\frac{i}{L}\left\{\frac{k k^{\prime}}{k+k^{\prime}}\left[\frac{\sin \left(k-k^{\prime}\right) L}{k-k^{\prime}} \cos \delta_{k}-\frac{\left(1-\cos \left(k-k^{\prime}\right) L\right)}{k-k^{\prime}} \sin \delta_{k}\right]\right. \\
& \left.+\frac{k k^{\prime}}{k-k^{\prime}}\left[\frac{\sin \left(k+k^{\prime}\right) L}{k+k^{\prime}} \cos \delta_{k}-\frac{\left(1-\cos \left(k+k^{\prime}\right) L\right)}{k+k^{\prime}} \sin \delta_{k}\right]\right\} .
\end{aligned}
$$

When we take the limit $L \rightarrow \infty$ in (4.8) and use that, since $g$ is purely imaginary, $g_{k k^{\prime}}=$ $-g_{k^{\prime} k}$, we have

$$
g_{k k^{\prime}}=-\frac{i}{L}\left\{\frac{\pi}{2} \delta\left(k^{2}-k^{\prime 2}\right) k k^{\prime}\left[\cos \delta_{k}-\cos \delta_{k^{\prime}}\right]-\frac{k k^{\prime}}{2} \mathcal{P}\left[\frac{\sin \delta_{k}+\sin \delta_{k^{\prime}}}{k^{2}-k^{\prime 2}}\right]\right\},
$$

with $\mathcal{P}$ meaning the principal value. This expression must now be used in the continuum version of (3.22) that reads 


$$
S\left(\omega, \omega^{\prime}\right)=\frac{L^{2}}{\pi^{2}} \int d k \int d k^{\prime}\left|g_{k k^{\prime}}\right|^{2} \delta\left(\omega-\Omega_{k}\right) \delta\left(\omega^{\prime}-\Omega_{k^{\prime}}\right)
$$

where $\Omega_{k}=\frac{\hbar^{2} k^{2}}{2 m}$. Evaluating (4.10) one gets

$$
S\left(\omega, \omega^{\prime}\right)=\frac{m}{8 \pi^{2} \hbar^{2}} \sqrt{\omega \omega^{\prime}}\left[\frac{\sin \delta(\omega)-\sin \delta\left(\omega^{\prime}\right)}{\omega-\omega^{\prime}}\right]^{2} .
$$

Now we can finally evaluate the integral for $\gamma(t)$ in (3.20) which in the long time approximation gives us

$$
\gamma(t)=\bar{\gamma}(T) \delta(t)
$$

where

$$
\bar{\gamma}(T)=-\frac{m}{2 \pi \hbar M} \int d E E R(E) \frac{d \bar{n}}{d E}
$$

is the damping constant that we want to study. In this expression the reflection coefficient $R(E)$ can be easily obtained from (4.5) as,

$$
R(E)=\frac{E_{0}}{E_{0}+E}
$$

where $E_{0}=\frac{m V_{0}^{2} a^{2}}{2 \hbar^{2}}$ is what we shall be calling the strength of the delta repulsive potential.

Let us now study (4.13) for fermions and bosons at zero and finite temperatures.

\section{A. Fermions}

At zero temperature it is straightfoward to write $\bar{\gamma}$ for the fermionic case. As we know, at $T=0$,

$$
\frac{d \bar{n}}{d E}=-\delta\left(E-E_{F}\right)
$$

where $E_{F}$ stands for the Fermi energy of the system. Therefore,

$$
\bar{\gamma}(T=0)=\frac{m}{2 \pi \hbar M} \frac{E_{0} E_{F}}{E_{0}+E_{F}} .
$$

Equation (4.16) tell us that for strong interactions or low densities $\left(E_{F}<<E_{0}\right)$ the damping constant is dominated by $E_{F}$. Although the reflection coefficent tends to one at 
very low energies, the number of fermions being scattered tends to zero. On the other hand, for weak interactions or high densities $\left(E_{F}>>E_{0}\right)$ the expression for the damping constant is governed by $E_{0}$ because at very high energies the reflection coefficient is vanishingly small and, consequently, all the scattering effects come from the low energy fermions $\left(E \leq E_{0}\right)$.

The finite temperature behavior of (4.13) will now be studied in two limits; the low and high temperature regimes. For studying the low temperature limit we make use of the well known Sommerfeld expansion [24] which allows us to write

$$
\int_{0}^{\infty} d E f(E)\left(-\frac{d \bar{n}}{d E}\right) \approx f(\mu)+\frac{\pi^{2}}{12} \frac{d^{2} f(\mu)}{d \mu^{2}}\left(k_{B} T\right)^{2}+\mathcal{O}\left(T^{4}\right)
$$

where, for the one dimensional Fermi gas,

$$
\mu \approx E_{F}+\frac{\pi^{2}}{12} \frac{\left(k_{B} T\right)^{2}}{E_{F}}
$$

Using (4.14) and (4.17) in (4.13) and keeping only terms $\mathcal{O}\left(T^{2}\right)$ we have

$$
\begin{aligned}
\bar{\gamma}(T) & =\frac{m E_{0}}{2 \pi \hbar M} \frac{x}{1+x}\left\{1+\frac{\pi^{2}}{12} \frac{1-x}{(1+x)^{2}}\left(\frac{k_{B} T}{E_{F}}\right)^{2}\right\} \\
x & =\frac{E_{F}}{E_{0}} .
\end{aligned}
$$

From (4.19) we see that the finite temperature correction to $\bar{\gamma}$ is strongly dependent on $x$. For strong interactions or low densities $(x<1)$ the low temperature correction to $\bar{\gamma}$ is positive. This means that we are starting to populate states with $E>E_{F}$ in a region where the reflection coefficient is still appreciable.

For weak potentials or high densities $(x>1)$ we have already seen that the damping is dominated by low energy states. As we increase the temperature there is no change in the behavior of the high energy fermions because of the very small values of the reflection coefficient in this region and only the depletion of the low energy states will influence the behavior of the damping constant lowering its zero temperature value.

The classical limit, which we define as $k_{B} T>>E_{F}$, can also be analyzed if we use that in this limit the Fermi occupation number is the same as in the distinguishable particle case, namely [17]; 


$$
\bar{n}(E)=\frac{\lambda}{g} \sqrt{\frac{2 \pi \hbar^{2} \beta}{m}} e^{-\beta E}
$$

where $g$ is the degeneracy factor and $\lambda$ the linear density of fermions,

$$
\lambda=\frac{N}{2 L}=\frac{g k_{F}}{\pi}
$$

Using (4.20) and (4.13) we can approximately evaluate that integral in two limits of interest,

$$
\begin{aligned}
\bar{\gamma}(T) & =\frac{m}{\pi M \hbar} \sqrt{\frac{k_{B} T E_{F}}{\pi}} & & E_{0}>>k_{B} T \\
\bar{\gamma}(T) & =\frac{m}{\pi M \hbar} \sqrt{\frac{E_{F}^{3}}{\pi k_{B} T}} & E_{0} & <k_{B} T .
\end{aligned}
$$

Actually there are three distinct cases which we can analyze in the whole temperature range.

a) $E_{F}>E_{0}$; in this case $\bar{\gamma}(0)$ is proportional to $E_{0}$ and starts to decrease as the temperature is raised. It keeps on decreasing up to high temperatures $\left(k_{B} T>>E_{F}>>E_{0}\right)$ where it has a $T^{-1 / 2}$ behavior.

b) $E_{0}>>E_{F}$; now $\bar{\gamma}(0)$ is proportional to $E_{F}$ and increases with temperature. However, there must be a maximum value for $\bar{\gamma}(T)$ since its predicted behavior at high temperatures $\left(k_{B} T>>E_{0}>>E_{F}\right)$ also obeys the $T^{-1 / 2}$ law.

c) Impenetrable external potential, $E_{0} \rightarrow \infty$; at low temperatures the behavior of the damping constant is exactly the same as the previous case. However, as the temperature increases it will never get to the $T^{-1 / 2}$ behavior because $E_{0}>>k_{B} T>>E_{F}$. Therefore it presents a $T^{1 / 2}$ for high temperatures.

The $T^{1 / 2}$ behavior of a) and b) above is easily understood because in both cases the energy $k_{B} T$ exceeds the strength $E_{0}$ of the potential barrier causing a strong depletion of the states which are sensitive to the presence of the scattering center.

For the impenetrable potential the reflection coefficient is always one and therefore $\bar{\gamma}(T)$ is a monotonically increasing function of $T$ and behaves as $T^{1 / 2}$ in the classical limit. These temperature dependences are illustrated in Fig.(1) and Fig.(2). 


\section{FIGURES}

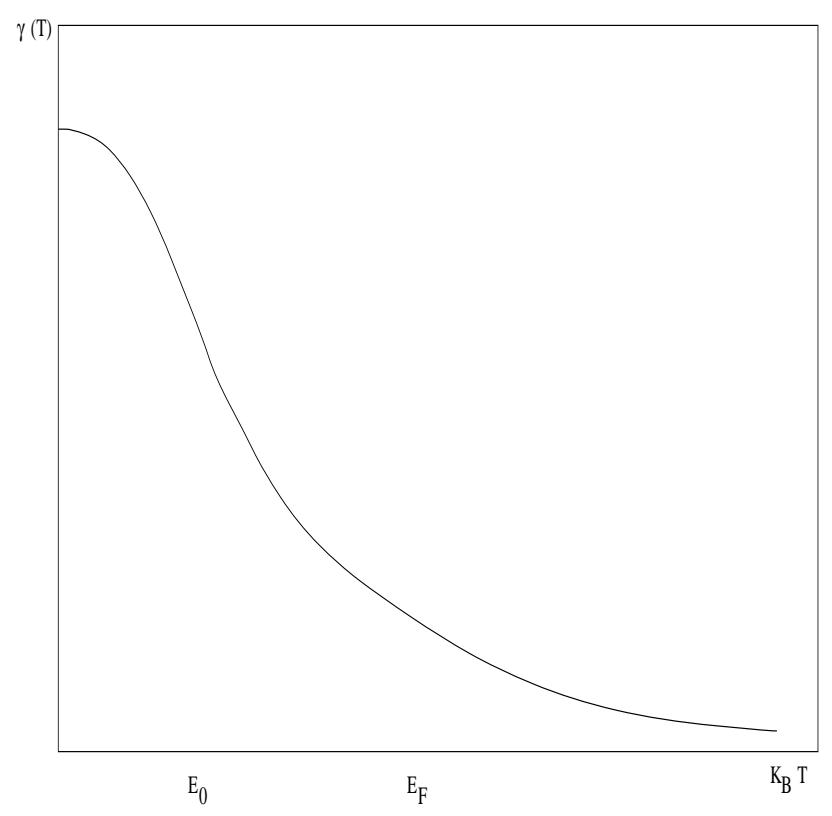

FIG. 1. Damping constant $\gamma(T)$ as a function of temperature for $E_{0}<E_{F}$

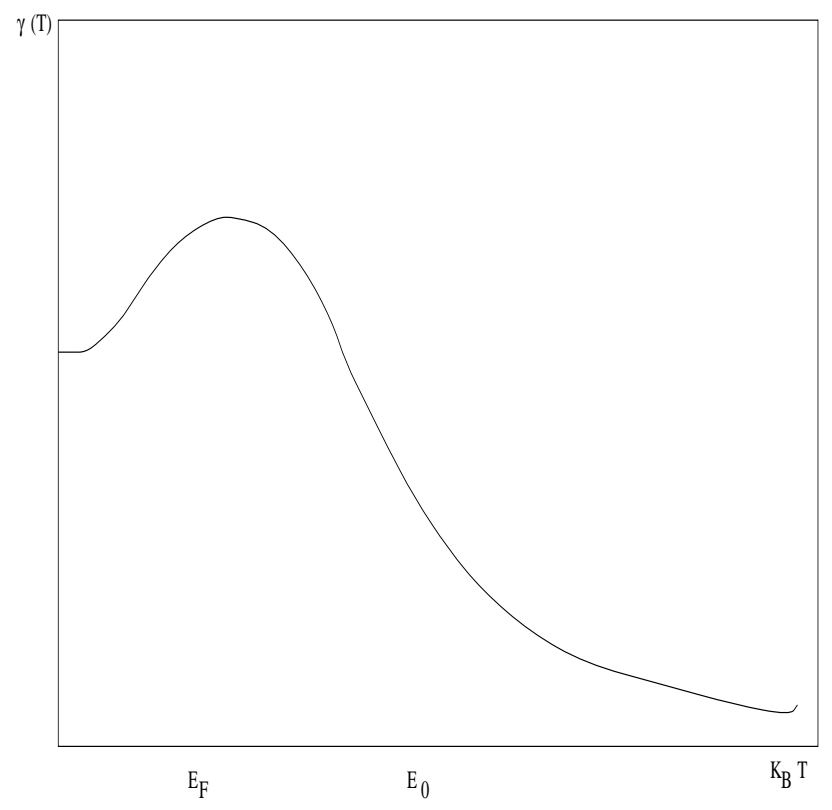

FIG. 2. Damping constant $\gamma(T)$ as a function of temperature for $E_{0}>E_{F}$ 


\section{B. Bosons}

The same analysis can be carried on in the case of massive bosons. The steps are not so straighforward as in the fermionic case but they present no special difficulty either. It is a very easy task to show that the occupation number for bosons has the following assymptotic limits;

$$
\begin{array}{ll}
\bar{n}(E)=\frac{k_{B} T}{E+|\mu|} & E+|\mu|<<k_{B} T \\
\bar{n}(E)=e^{-\frac{E+|| \mid}{k_{B} T}} & E+|\mu|>>k_{B} T .
\end{array}
$$

Observe that for bosons the chemical potential $\mu$ is always negative [17].

Now, if we assume that, when $T \rightarrow 0, \mu(T)$ behaves as 25

$$
\mu(T)=-\frac{k_{B} T}{N}
$$

we can deduce from (4.23) that

$$
\bar{n}(E=0)=N
$$

where $N$ ist the total number of bosons in the system.

In order to further investigate the crossover from the low to high energy behavior of $\bar{n}(E)$ in (4.23-4.24) let us assume that we are in the high temperature regime which means that we can replace the chemical potential by its one dimensional classical expression [17],

$$
\mu_{1 D}(T) \approx \frac{k_{B} T}{2} \ln \left(\frac{T_{0}}{T}\right)
$$

where $T_{0}=\frac{2 \pi \hbar^{2} \lambda^{2}}{m k_{B}}$ is the temperature to localize a particle in a region of length $2 L / N$ and $\lambda$ is, as in (4.21), the linear density of particles. Notice that by high temperatures we mean $T>T_{0}$. In this case the crossover takes place at the energy

$$
E_{c}=k_{B} T-|\mu|=k_{B} T\left[1+\frac{1}{2} \ln \left(\frac{T_{0}}{T}\right)\right] .
$$

Since $\ln \left(T_{0} / T\right)$ is a monotonically decreasing function of $T$ we see that as we increase $T$ the crossover energy $E_{c}$ is pulled down to lower values and eventually reaches zero. This means 
that at this temperature the occupation number can be treated classically for any energy $E$. It is a trivial matter to calculate this temperature because when is is reached one has $E_{c}\left(T_{c}\right)=0$ or

$$
T_{c} \approx e^{2} T_{0}
$$

Therefore, this is the temperature above which the system is classical.

The same analysis tells us that when $T<<T_{0}$ the crossover energy $E_{c}$ is very high and, consequently, the low energy form of $\bar{n}(E)$ dominates any physical phenomenon in this temperature range. Actually we can use (4.23) to compute $N_{0}(T)$, the number of particles in the lowest energy state, for very low $T$. We can do this by writing that the total number of bosons can be approximated by the expression

$$
N \approx \frac{L}{\pi} \sqrt{\frac{2 m}{\hbar^{2}}} \int_{0}^{k_{B} T-|\mu|} \frac{d E}{2 \sqrt{E}} \frac{k_{B} T}{E+|\mu|}=\frac{L}{\pi} \sqrt{\frac{2 m}{\hbar^{2}}} \frac{k_{B} T}{\sqrt{|\mu|}} \tan ^{-1} \sqrt{\frac{k_{B} T-|\mu|}{|\mu|}}
$$

and then, assuming that

$$
\operatorname{Lim}_{N \rightarrow \infty, T \rightarrow 0} \frac{|\mu|}{T}=0
$$

which is in agreement with (4.24), one gets

$$
\mu(T) \approx-\frac{k_{B} T}{N_{0}(T)}
$$

where $N_{0}(T) \approx \frac{4 T_{0}}{\pi T}$.

This is the result that enable us to compute the low temperature behavior of $\bar{\gamma}(T)$. This is done by integrating (4.13) by parts and using (4.23) to write

$$
\bar{\gamma}(T)=\frac{m}{2 \pi \hbar M} \int_{0}^{k_{B} T-|\mu|} d E\left(\frac{E_{0}}{E+E_{0}}\right)^{2} \frac{k_{B} T}{E+|\mu|}
$$

which with the help of (4.31) yields

$$
\bar{\gamma}(T)=\frac{m}{2 \pi \hbar M} k_{B} T \ln \left(\frac{4 T_{0}}{\pi T}\right)
$$

From this formula we see that the damping constant vanishes at $T=0$ but presents a non-analytic behavior as a function of temperature in this limit. This is due to the fact 
that $\bar{n}(E)$ is proportional to $\sqrt{E} \delta(E)$ when $T \rightarrow 0$ and therefore the weight of the states influenced by the external particle vanishes in this limit. As the temperature is raised there is a fast increase of this weight.

The high temperature limit of the damping constant has the same form as for the fermionic case (as it should) with the only difference that $E_{F}$ must now be replaced by $k_{B} T_{0}$. Then,

$$
\begin{array}{rr}
\bar{\gamma}(T)=\frac{m k_{B}}{\pi M \hbar} \sqrt{\frac{T T_{0}}{\pi}} & E_{0}>>k_{B} T \\
\bar{\gamma}(T)=\frac{m}{\pi M \hbar} \sqrt{\frac{\left(k_{B} T_{0}\right)^{3}}{\pi k_{B} T}} & E_{0}<<k_{B} T .
\end{array}
$$

Once again following our procedure in the fermionic case we can also study distinct temperature dependences of the damping constant as we show below.

a) $E_{0}>k_{B} T$; here $\bar{\gamma}$ vanishes at $T=0$ and increases with temperature as in (4.33). When $k_{B} T>>E_{0}>>k_{B} T_{0}$ it decays as $T^{-1 / 2}$ and, consequently, it must present a maximum at intermediate temperatures $\left(E_{0}>>k_{B} T>>k_{B} T_{0}\right)$.

b) $E_{0} \rightarrow \infty$; the low temperature behaviour is the same as before but now one can never go beyond the regime $E_{0}>>k_{B} T>>k_{B} T_{0}$ and therefore $\bar{\gamma}$ behaves like $T^{1 / 2}$.

Notice that we have not analyzed above the condition $k_{B} T_{0}>>E_{0}$ because it does not make sense for bosons. In this case the strength of the barrier is below the minumum energy required to localize a boson within $2 L / N$ and so this would correspond to an undamped motion.

The interpretation of the high temperature behavior of $\bar{\gamma}$ follows similarly to what we have said for fermions.

Here we can also scketch the behavior of $\bar{\gamma}$ for a fixed $T_{0}$ as we show in Fig.(3). 


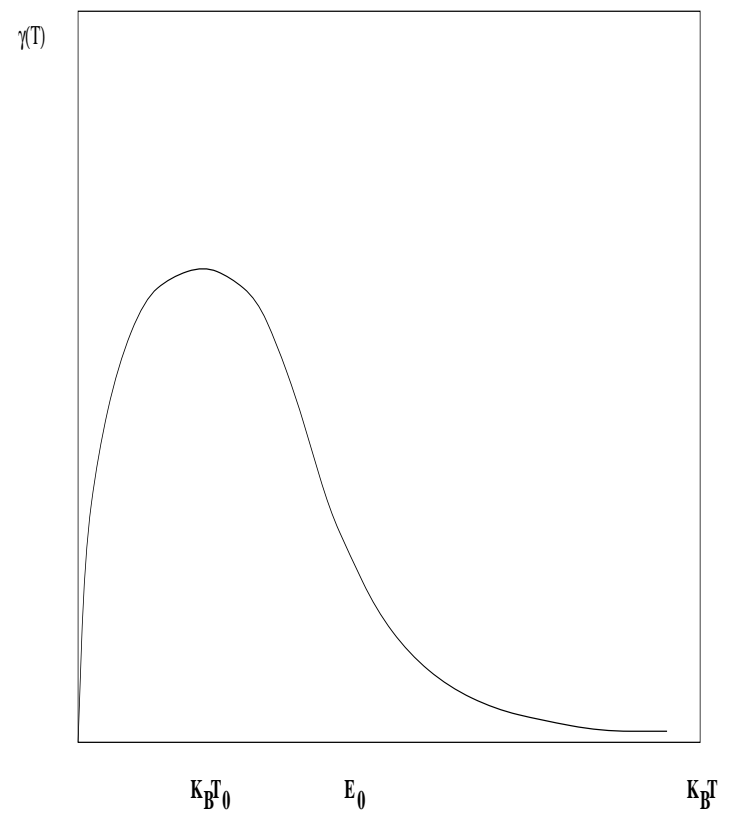

FIG. 3. Damping constant $\gamma(T)$ as a function of temperature for $k_{B} T_{0}<E_{0}$

\section{CONCLUSIONS}

In this paper we succeeded in writing down an expression for the damping constant of a particle coupled via a delta repulsive potential to non-interacting bosons and fermions.

We were able to show that using a very simple unitary transformation we could map the original hamiltonian into a new one where the external particle interacts via a momentummomentum coupling to a new system where the particles are acted by an external potential. Moreover, we could also show that within the Feynman-Vernon picture all we needed was to find the time evolution of the reduced density operator for the transformed system.

Although our results were quite general very little can be done beyond the gaussian approximation of the influence functional. On the other hand this is exactly the form we should have got had we started with a bath of harmonic oscillators [1,2] and therefore, a question still remains; why haven't we started with this simpler model?

The answer is simple. In our present problem our input is the microscopic parameters of the interaction while in [1,2] all one has is a constraint to recover the Langevin equation in the classical limit. There, the microscopic constants all combine in order to establish the 
low frequency behavior of the spectral function $J(\omega)$ [1, 2] to be the form $\eta \omega$, where $\eta$ is the damping constant, whereas in the present approach the damping constant is obtained as a function of the microscopic parameters and the temperature. It is clear one could try to find a temperature dependent spectral function $J(\omega, T)$ in order to recover our function $\bar{\gamma}(T)$. However, it is hard to imagine that this could be done a priori, without the knowledge of the specific form of $\bar{\gamma}(T)$. Even if we can phenomenologically describe $\bar{\gamma}(T)$ in a vast temperature range it is many times of great importance to understand this behavior from first principles.

Notice that in the present formulation we have also a prescription to go beyond the gaussian approximation. This can be used if one wants to test the stability of the "mean field" solution to this problem. This project is being carried forward by one of us in a separate contribution [26].

\section{ACKNOWLEDGMENTS}

We are deeply indebt to H.Castella, A.O.Gogolin, M.P.A.Fisher, A.W.W.Ludwig, N.Prokofev and X. Zotos for many useful comments and discussions. A.O.C. is also grateful to the partial support of the Conselho Nacional de Desenvolvimento Científico e Tecnológico (CNPq-Brazil). This work was partially supported by the NSF through grants DMR92-14236 and PHY89-04035. 


\section{APPENDIX A: FORMULATION IN THERMAL EQUILIBRIUM}

In this appendix we extend the real time calculation of the paper to imaginary time and stress the approximations used in the bulk of the paper.

We look here at the partition function for the initial problem which can be written as,

$$
Z=\operatorname{tr}\left(e^{-\beta H}\right)=\operatorname{tr}\left(e^{-\beta H^{\prime}}\right)
$$

where we have used the unitary transformation (2.3) and the fact that the trace is invariant under this transformation.

The partition function can be written in terms of path integrals for the external particle and the fields of the bath as

$$
Z=\int D q \int D \Psi^{*} D \Psi \exp \left(-S_{E}\left[q, \Psi^{*}, \Psi\right]\right)
$$

where the action associated with the hamiltonian $(2.5)$ reads $(\hbar=1)$,

$$
S_{E}=\int_{0}^{\beta} d t\left\{\frac{M \dot{x}^{2}}{2}-i \dot{x} \sum_{k, k^{\prime}} g_{k k^{\prime}} \Psi_{k}^{*} \Psi_{k^{\prime}}-\sum_{k}\left(\frac{d}{d t}-E_{k}\right) \Psi_{k}^{*} \Psi_{k}\right\} .
$$

Since we are interested only in the dynamics of the particle we can trace the environmental particles exactly in order to get,

$$
Z=\int D q \exp \left\{-S_{e f f}[q]\right\}
$$

where the effective action for the particle is non-linear and reads

$$
S_{e f f}=\int_{0}^{\beta} d t \frac{M \dot{x}^{2}}{2} \mp \ln \operatorname{det} W[q]
$$

where the minus sign is for fermions and the plus sign for bosons. The operator $W$ is written in terms of its matrix elements as,

$$
\left\langle k t|W| k^{\prime} t^{\prime}\right\rangle=\delta\left(t-t^{\prime}\right)\left[\left(\frac{d}{d t}-E_{k}\right) \delta_{k k^{\prime}}-i \dot{x} g_{k k^{\prime}}\right] .
$$

Clearly the problem (A5) is extremely complicated and is equivalent to the one obtained in real time from expression (3.10). However we are interested only in the case where the 
velocity of the particle is small or its mass is much larger than the mass of the environmental particles. Therefore, we look at the saddle point equations for the action (A5) in the limit where $M \rightarrow \infty$. It is very easy to show that within the mean field approximation the saddle point equations read

$$
\ddot{x} \pm \frac{i}{M} \sum_{k, k^{\prime}} g_{k k^{\prime}}\left(\frac{d}{d t^{\prime}} G\left(k, t ; k^{\prime}, t^{\prime}\right)\right)_{t^{\prime}=t}=0
$$

and

$$
\left(\frac{d}{d t}-E_{k}\right) G\left(k, t ; k^{\prime}, t^{\prime}\right) \mp i \dot{x}(t) \sum_{k^{\prime \prime}} g_{k k^{\prime \prime}} G\left(k^{\prime \prime}, t ; k^{\prime}, t^{\prime}\right)=\delta_{k k^{\prime}} \delta\left(t-t^{\prime}\right)
$$

In the limit $M \rightarrow \infty$ we choose the particular solution

$$
\begin{aligned}
x(t) & =0 \\
\left(\frac{d}{d t}-E_{k}\right) G_{0}\left(k, t ; k^{\prime}, t^{\prime}\right) & =\delta_{k k^{\prime}} \delta\left(t-t^{\prime}\right)
\end{aligned}
$$

which can be easily solved by

$$
G_{0}\left(k, t ; k^{\prime}, t^{\prime}\right)=\frac{\delta_{k, k^{\prime}}}{\beta} \sum_{n} \frac{e^{i \omega_{n}\left(t-t^{\prime}\right)}}{i \omega_{n}-E_{k}}
$$

where $\omega_{n}=\frac{2 \pi n}{\beta}$ for bosons and $\omega_{n}=\frac{\pi(2 n+1)}{\beta}$ for fermions.

Now we look at gaussian fluctuations around this mean field by expanding the determinant in (A5) as

$$
\ln \operatorname{det} W=\ln \operatorname{det}\left(H_{0}+H_{I}\right) \approx \ln \operatorname{det} G_{0}^{-1}+\operatorname{tr}\left(G_{0} H_{I}\right)-\frac{1}{2} \operatorname{tr}\left(G_{0} H_{I}\right)^{2}
$$

where $H_{0} G_{0}=1$ and $H_{I}$ is the second term on the r.h.s. of (A6).

Thus, the effective action reads

$$
S_{e f f} \approx S_{e f f}^{0}+\int_{0}^{\beta} \frac{M \ddot{x}^{2}}{2}+\int_{0}^{\beta} d t \int_{0}^{\beta} d t^{\prime} F\left(t-t^{\prime}\right) \dot{x}(t) \dot{x}\left(t^{\prime}\right)
$$

where

$$
F(t)=\sum_{k, k^{\prime}}\left|g_{k k^{\prime}}\right|^{2} G_{0}(k, t) G_{0}\left(k^{\prime},-t\right)
$$


It is useful to consider the action (A12) in Matsubara representation [17]

$$
\Delta S_{\text {eff }}=\frac{1}{\beta} \sum_{n}\left(M \omega_{n}^{2}+\omega_{n}^{2} F\left(\omega_{n}\right)\right) x\left(\omega_{n}\right) x\left(-\omega_{n}\right)
$$

where

$$
F\left(\omega_{n}\right)=\sum_{k, k^{\prime}} \frac{\left|g_{k, k^{\prime}}\right|^{2}}{i \omega_{n}+E_{k}-E_{k^{\prime}}}\left(\bar{n}_{k}-\bar{n}_{k^{\prime}}\right) .
$$

The actual expression for $F\left(\omega_{n}\right)$ now depends on the form of the coupling constant $g_{k k^{\prime}}$. In the paper we considered the case of a delta repulsive interaction but in the following subsection we will consider the case of a general potential without bound states. This approach generalizes the results of section IV.

\section{Matrix element}

Let us consider the case where the potential of interaction between the particle and the environmental particles is localized, that is, $V(x)=0$ for $x>|a|$. In this case the Schrödinger equation for the impurity problem in the assymptotic limit can be written as

$$
\frac{d^{2}}{d x^{2}} \Psi_{k}(x)+k^{2} \Psi_{k}(x)=0
$$

where $E_{k}=\frac{k^{2}}{2 m}$.

The general solution of this problem can be written in terms of even and odd wavefunctions as follows [23];

$$
\begin{aligned}
& \Psi_{k}^{E}(x)=\sqrt{\frac{2}{L}} \cos \left(k|x|+\delta_{E}(k)\right) \\
& \Psi_{k}^{O}(x)=\sqrt{\frac{2}{L}} \operatorname{sgn}(x) \sin \left(k|x|+\delta_{O}(k)\right)
\end{aligned}
$$

where $\operatorname{sgn}(x)=+1(-1)$ if $x>0(x<0)$, and $\delta_{E}(k)$ and $\delta_{O}(k)$ are the even and odd phase shifts, respectively.

The phase shifts are related to the reflection and transmission coefficients by a simple relation 


$$
\begin{aligned}
& r(k)=\frac{e^{2 i \delta_{E}(k)}-e^{2 i \delta_{O}(k)}}{2} \\
& t(k)=\frac{e^{2 i \delta_{E}(k)}+e^{2 i \delta_{O}(k)}}{2},
\end{aligned}
$$

which can be inverted in order to give

$$
\begin{aligned}
& \delta_{E}(k)=\frac{1}{2} \tan ^{-1}\left(\frac{\operatorname{Im}(t(k)+r(k))}{\operatorname{Re}(t(k)+r(k))}\right) \\
& \delta_{O}(k)=\frac{1}{2} \tan ^{-1}\left(\frac{\operatorname{Im}(t(k)-r(k))}{\operatorname{Re}(t(k)-r(k))}\right) .
\end{aligned}
$$

The reflection and transmission coefficients can be now expressed in terms of the phase shifts as

$$
\begin{gathered}
R(k)=|r(k)|^{2}=\sin ^{2}\left(\delta_{E}(k)-\delta_{O}(k)\right) \\
T(k)=|t(k)|^{2}=\cos ^{2}\left(\delta_{E}(k)-\delta_{O}(k)\right) .
\end{gathered}
$$

Thus, any information from the scattering process is encapsulated in the phase shifts.

From (3.1) it is easy to see that the coupling constant $g_{k k^{\prime}}$ only couples states with different parities, that is,

$$
\begin{gathered}
g_{k k^{\prime}}^{O, O}=g_{k k^{\prime}}^{E, E}=0 \\
g_{k k^{\prime}}^{O, E}=-g_{k^{\prime} k}^{E, O}
\end{gathered}
$$

The calculation of the matrix element follow the same steps as in (4.7) and (4.8) and reads

$$
g_{k k^{\prime}}^{O, E}=\frac{i}{2 L} \frac{k k^{\prime}}{k+k^{\prime}} \mathcal{P}\left(\frac{\sin \left(\delta_{O}(k)-\delta_{E}\left(k^{\prime}\right)\right)+\sin \left(\delta_{O}\left(k^{\prime}\right)-\delta_{E}(k)\right)}{k-k^{\prime}}\right) .
$$

The integral we need to evaluate in (A15) can now be written as

$$
F\left(\omega_{n}\right)=\frac{1}{4 L^{2}} \sum_{k, k^{\prime}} \frac{\left(\sin \left(\delta_{O}(k)-\delta_{E}\left(k^{\prime}\right)\right)+\sin \left(\delta_{O}\left(k^{\prime}\right)-\delta_{E}(k)\right)\right)^{2}\left(\bar{n}_{k}-\bar{n}_{k^{\prime}}\right)}{\left(k^{2}-\left(k^{\prime}\right)^{2}\right)^{2}\left(E_{k}-E_{k^{\prime}}+i \omega_{n}\right)} .
$$

This integral can be done by choosing a convenient countor in the complex plane which avoids the double pole. The final result is

$$
F\left(\omega_{n}\right)=\frac{1}{L\left|\omega_{n}\right|} \sum_{p} p^{2} R(p)\left(-\frac{d \bar{n}_{p}}{d p}\right)
$$


Thus, from (A24) and (A14), the effective action reads

$$
\Delta S_{e f f}=\frac{M}{\beta} \sum_{n}\left(\omega_{n}^{2}+\gamma\left|\omega_{n}\right|\right) x\left(\omega_{n}\right) x\left(-\omega_{n}\right)
$$

which is the usual action for the motion of a particle in a dissipative environment [2] with a damping constant

$$
\gamma=\frac{1}{M L} \sum_{p} p^{2} R(p)\left(-\frac{d \bar{n}_{p}}{d p}\right)
$$

Equation (A26) can be rewritten using the usual definition of the density of states

$$
D(E)=\frac{1}{L} \sum_{p} \delta\left(E-E_{p}\right)
$$

as

$$
\gamma=\frac{m}{M} \int_{-\infty}^{\infty} d E D(E) E R(E) v(E)\left(-\frac{d \bar{n}(E)}{d E}\right)
$$

where $p^{2}=2 m E$ and $v(E)=\frac{d E}{d p}$. It turns out that in one dimension the density of states is simply

$$
D(E)=\frac{1}{2 \pi v(E)}
$$

and (A30) becomes identical to (4.13). 
* On leave of absence from Instituto de Física "Gleb Wataghin", Departamento de Física do Estado Sólido e Ciência dos Materiais, Universidade Estadual de Campinas, 13083-970, Campinas, SP, Brazil. 


\section{REFERENCES}

[1] A.O.Caldeira and A.J.Leggett, Physica 121 A, 587 (1983).

[2] A.O.Caldeira and A.J.Leggett, Ann.Phys.149, 347 (1983).

[3] A.J.Leggett, S.Chakravarty, A.T.Dorsey, M.P.A.Fisher, A.Garg, and W.Zwerger, Rev.Mod.Phys. 59, 1, (1987).

[4] N.V.Prokofev, Int.Jour.Mod.Phys. B 7, 3327 (1993) and references therein.

[5] See, for example, J.Kurkijärvi and A.L.Fetter, A.I.Ahonen et al, T.Soda or P.D.Roach, J.B.Ketterson and P.R.Roach, all in Quantum Fluids and Solids ed. by S.B.Trickey, E.D.Adams and J.W.Dufty (1977, Plenum Press, NY).

[6] J.Kondo, Physica 84 B, 40 (1976); J.Kondo and J.Soda, J.Low Temp.Phys. 50, 21 (1983); J.Kondo, Physica 126 B, 377 (1984).

[7] K.Yamada, Prog.Th.Phys. 72, 195 (1984); K.Yamada, A.Sakurai and S.Miyazima, Prog.Th.Phys 73, 1342 (1985).

[8] P.Hedegard and A.O.Caldeira, Phys.Scripta 35, 609 (1987); Phys.Rev.B 35, 106 (1987).

[9] C.W.Clawson et al, Phys.Rev.Lett 51, 114 (1983).

[10] A.H.Castro Neto and A.O.Caldeira, Phys.Rev.B 46, 8858 (1992).

[11] A.H.Castro Neto and A.O.Caldeira, Phys.Rev.E 48, 13974 (1993).

[12] See, for example, R.Rajaraman, Solitons ans Instantons (North Holland, Amsterdam, 1992) and references therein.

[13] A.H.Castro Neto and A.O.Caldeira, Phys.Rev.Lett. 67, 1960 (1991).

[14] R.P.Feynman and F.L.Vernon, Ann.Phys (NY) 24, 118 (1963).

[15] For another application of the same unitary transformation see V.Hakim and V.Ambegaokar, Phys.Rev.A 32, 423 (1985). 
[16] H.Castella and X.Zotos, Phys.Rev.B 47, 16186 (1993).

[17] See, for instance, A.L.Fetter and J.D.Walecka, Quantum Theory of Many Particle Systems (McGraw-Hill, 1971).

[18] See, for instance, J.W.Negele and H.Orland, Quantum Many Particle Systems (AddisonWesley, 1988).

[19] Notice that expression (4.27) in ref. [10] is not entirely correct. However, due to the gaussian approximation performed later in eq. (4.32) the expression for the damping function remains unchanged whereas the diffusion constant is only slightly altered in the high temperature limit. Their correct expressions are (3.20) and (3.21) of the present paper.

[20] C. L. Kane, M. P. A. Fisher and J. Polchinski, Phys. Rev. Lett. 72, 4129, (1994).

[21] A.O.Gogolin, Ann.Phys.(Fr) 19, 241 (1994)

[22] P.Fendley, A.W.W. Ludwig and H.Saleur, unpublished; A.M.Tsvelik, unpublished.

[23] H.J.Lipkin, Quantum Mechanics: New Approaches to Selected Topics (North-Holland, Amsterdam, 1973).

[24] See, for example, N.W.Ashcroft and N.D.Mermim, Solid State Physics (Holt,Rindmart and Winston, 1976).

[25] R.P.Feynman, Statistical Mechanics (W.A.Benjamin, 1972).

[26] A.H.Castro Neto and M.P.A.Fisher, in preparation. 